\title{
TENSOR CATEGORIES AND VACANT DOUBLE GROUPOIDS
}

\author{
Juan Martín Mombelli and Sonia Natale
}

\begin{abstract}
We show that fusion categories $\operatorname{Rep}\left(\mathbb{k}_{\tau}^{\sigma} \mathcal{T}\right)$ of representations of the weak Hopf algebra coming from a vacant double groupoid $\mathcal{T}$ and a pair $(\sigma, \tau)$ of compatible 2-cocyles are group-theoretical.
\end{abstract}

\section{Introduction}

Semisimple tensor categories appear encoding symmetries of distinct mathematical structures. This makes the problem of their classification both a highly interesting and difficult one. The problem is difficult even for special classes of these categories like representations of finite dimensional semisimple Hopf algebras. The main goal of this paper is to establish a relation between two recent constructions of semisimple tensor categories.

An important class of fusion categories was introduced by Ostrik in [O2], also studied in the paper [ENO], by Etingof, Nikshych and Ostrik. These fusion categories are built up from finite group data, and are called group-theoretical.

Let $D$ be a finite group and let $V \subseteq D$ be a subgroup. Let also $\omega \in Z^{3}\left(D, \mathbb{k}^{\times}\right)$be a normalized 3-cocycle, $\psi \in C^{2}\left(V, \mathbb{k}^{\times}\right)$a normalized 2-cochain, such that $\left.\omega\right|_{V \times V \times V}=$ $d \psi$. Denote by $\mathcal{C}(D, \omega)=\operatorname{Vect}_{\omega}^{D}$ the tensor category of finite dimensional $D$-graded vector spaces with associativity constraint given by the 3-cocycle $\omega$. The twisted group algebra $\mathbb{k}_{\psi} V$ is an algebra in this category. The category $\mathcal{C}(D, \omega, V, \psi)$ is defined to be the category of $\mathbb{k}_{\psi} V$-bimodules in Vect ${ }_{\omega}^{D}$. This is a fusion category with tensor product $\otimes_{\mathbb{k}_{\psi} V}$ and unit object $\mathbb{k}_{\psi} V$. A fusion category is called group-theoretical if it is equivalent to a category of the form $\mathcal{C}(D, \omega, V, \psi)$.

By the results in [ENO], the simple objects in a group theoretical category have integer Frobenius-Perron dimensions and thus every group theoretical category is the representation category of a semisimple finite dimensional quasi-Hopf algebra. An explicit description (up to gauge equivalence) of these quasi-Hopf algebras was given in [N2]: the quasi-Hopf algebras appearing in this description are a natural generalization of the Dijkgraaf-Pasquier-Roche twisted quantum doubles $D^{\omega} G$ [DPR]. It has been asked in [ENO] if group-theoretical Hopf algebras exhaust the class of semisimple Hopf algebras. The answer to this question is not known until now.

Another construction of a family of tensor categories arising from finite vacant double groupoids was done in [AN1], and later generalized to the nonvacant case in [AN2]. In these papers, a semisimple weak Hopf algebra $\mathbb{k}^{\vartheta} \mathcal{T}$ is naturally attached to a finite

Received by the editors September 2, 2005.

2000 Mathematics Subject Classification. 16W30.

This work was partially supported by Agencia Córdoba Ciencia, ANPCyT-Foncyt, CONICET, Fundación Antorchas and Secyt (UNC). 
double groupoid $\mathcal{T}$ satisfying a certain filling condition and a certain perturbation datum $\vartheta$, and giving a fortiori a semisimple tensor category of representations.

It is natural to ask if any fusion category arising from this construction is grouptheoretical. Several examples studied in [AN1] turn out to be group-theoretical. Necessary and sufficient conditions on the double groupoid $\mathcal{T}$ in order that the category $\operatorname{Rep} \mathbb{k} \mathcal{T}$ be fusion were given in [AN2]. It is also shown in [AN2] that in the cases when $\vartheta$ comes from 'corner functions' intrinsically attached to the double groupoid, the category $\operatorname{Rep} \mathbb{k}^{\vartheta} \mathcal{T}=\operatorname{Rep} \mathbb{k} \mathcal{T}$ is the representation category of a finite-dimensional semisimple quasi-Hopf algebra.

In this paper we show that the tensor categories of the form $\operatorname{Rep} \mathbb{k}_{\tau}^{\sigma} \mathcal{T}$, arising from a vacant double groupoid $\mathcal{T}$ and a pair of compatible cocycles $\sigma, \tau$ as in [AN1], are group-theoretical whenever they are fusion. See Theorems 5.2 and 5.3. The precise condition under which these categories are fusion has been given in [AN1]: it reduces to the connectedness of the groupoid of vertical edges. Our proof relies on the fact that vacant double groupoids are essentially the same as matched pairs of groupoids $[\mathrm{M}]$. This allows us to prove generalizations of certain category equivalences valid for finite groups, and get the result. We give an explicit, although not canonical, tensor equivalence between $\operatorname{Rep} \mathbb{k}_{\tau}^{\sigma} \mathcal{T}$ and a group-theoretical fusion category. One of the main difficulties we encounter is that, although in the connected case we may transfer 'group-theoretical facts' to 'groupoid-theoretical facts', some cohomological obstructions appear. This is evidenced in Subsection 2.4, c.f. Proposition 2.5 therein.

It follows from our results that the Drinfeld center of the category $\operatorname{Rep} \mathbb{k}_{\tau}^{\sigma} \mathcal{T}$ is equivalent to the representation category of a twisted quantum double [DPR]. In view of the description in [N2] of group-theoretical quasi-Hopf algebras, our results in the vacant context also bring implicit a description of a quasi-Hopf algebra whose representation category is equivalent to $\operatorname{Rep} \mathbb{k}_{\tau}^{\sigma} \mathcal{T}$.

The paper is organized as follows: in Section 2 we study several examples of tensor categories arising from finite groupoids, which generalize group-theoretical categories. In Section 3 we recall the definition and characterizations of vacant double groupoids. Section 4 contains the construction of the associated weak Hopf algebras from [AN1]; we also give in this section an alternative description of the corresponding tensor category of representations in terms of matched pairs of groupoids. Finally, in Section 5 we prove our main result.

Acknowledgement. The question we consider in this paper arose from discussions with N. Andruskiewitsch. Both authors thank him for encouraging.

Conventions and notation. We shall work over an algebraically closed field $\mathbb{k}$ of characteristic zero. The group of units of $\mathbb{k}$ is denoted by $\mathbb{k}^{\times}$. All vector spaces and algebras are assumed to be over $\mathbb{k}$.

A groupoid with source $\mathfrak{s}$ and target $\mathfrak{e}$ will be denoted as a diagram $\mathfrak{e}, \mathfrak{s}: \mathcal{D} \rightrightarrows \mathcal{P}$. When $h, g \in \mathcal{D}$ are such that $\mathfrak{e}(h)=\mathfrak{s}(g)$ their composition will be indicated by $h g$. We shall identify the base $\mathcal{P}$ with a subset of $\mathcal{D}$ via the identity map: $P \mapsto \mathrm{id}_{P} \in \mathcal{D}$.

We shall say that the groupoid $\mathcal{D} \rightrightarrows \mathcal{P}$ is finite if $\mathcal{D}$ (hence also $\mathcal{P}$ ) is a finite set. The set of arrows $\alpha$ with source $\mathfrak{s}(\alpha)=P$ and target $\mathfrak{e}(\alpha)=Q$ will be indicated $\mathcal{D}(P, Q)$; thus $\mathcal{D}(P, Q)$ is a torsor over the group $\mathcal{D}(P)=\mathcal{D}(P, P)$. The groupoid 
$\mathcal{D} \rightrightarrows \mathcal{P}$ defines an equivalence relation $\sim$ on $\mathcal{P}$ by declaring $P \sim Q$ if and only if $\mathcal{D}(P, Q) \neq \emptyset$. We shall say that the groupoid is connected if this relation is connected.

If $\mathcal{D} \rightrightarrows \mathcal{P}$ is connected, then it is isomorphic to the direct product $\mathcal{D}(P) \times \mathcal{P}^{2}$, where $P$ is any element of $\mathcal{P}$. In general, let $X$ be an equivalence class of $\sim$ and let $\mathcal{D}_{X}$ be corresponding connected groupoid on the base $X$ induced by $\mathcal{D}$. Then $\mathcal{D} \simeq \coprod_{X \in \mathcal{P} / \sim} \mathcal{D}_{X}$.

\section{Tensor categories coming from finite groupoids}

2.1. Preliminaries on tensor categories. The reader is referred to [O1] for an exposition of the notions of tensor categories and their module categories used throughout in this paper.

We shall be interested in a special class of tensor categories, whose definition we recall next. These type of categories have been intensively studied by a number of people in the last years.

Definition 2.1. A fusion category over $\mathbb{k}$ is a $\mathbb{k}$-linear semisimple and rigid tensor category $\mathcal{C}$ such that:

(i) all hom spaces are finite dimensional;

(ii) the set of isomorphism classes of simple objects in $\mathcal{C}$ is finite;

(iii) the unit object 1 is simple.

If $\mathcal{C}$ satisfies (i) and (ii), then $\mathcal{C}$ is called a multi-fusion category. See [ENO].

Suppose that $\mathcal{C}$ is a multi-fusion category. Write $\mathbf{1}=\oplus_{i} \mathbf{1}_{i}$, where $\mathbf{1}_{i}$ are the simple constituents of the unit object $\mathbf{1}$. Then $\mathcal{C}=\oplus_{i, j} \mathcal{C}_{i j}$, where $\mathcal{C}_{i j}$ is the full subcategory whose objects $X$ satisfy $X \otimes \mathbf{1}_{j}=X=\mathbf{1}_{i} \otimes X$. The categories $\mathcal{C}_{i i}$ are fusion categories called the fusion components of $\mathcal{C}$.

Assume $\mathcal{C}$ is indecomposable. Then $\mathcal{M}=\oplus_{j} \mathcal{C}_{j i}$ is an indecomposable module category over $\mathcal{C}[\mathrm{O} 1]$, and there are isomorphisms [ENO, 2.4]

$$
\mathcal{C}_{\mathcal{M}}^{*}=\operatorname{Fun}_{\mathcal{C}}(\mathcal{M}, \mathcal{M}) \simeq \mathcal{C}_{i i}^{\mathrm{op}}
$$

For the rest of this section we fix a finite groupoid $\mathfrak{e}, \mathfrak{s}: \mathcal{D} \rightrightarrows \mathcal{P}$ and let $\omega \in$ $Z^{3}\left(\mathcal{D}, \mathbb{k}^{\times}\right)$be a normalized 3-cocycle; that is, $\omega: \mathcal{D}_{\mathfrak{e}} \times{ }_{\mathfrak{s}} \mathcal{D}_{\mathfrak{e}} \times{ }_{\mathfrak{s}} \mathcal{D} \rightarrow \mathbb{k}^{\times}$, such that

$$
\begin{aligned}
\omega(a, b, c) \omega(a, b c, d) \omega(b, c, d) & =\omega(a b, c, d) \omega(a, b, c d), \\
\omega(a, P, b) & =1,
\end{aligned}
$$

for all appropriately composable arrows $a, b, c, d \in \mathcal{D}, P \in \mathcal{P}$.

2.2. Pointed multi-fusion categories. Denote by $\mathcal{C}(\mathcal{D}, \omega)$ the category of finite dimensional $\mathcal{D}$-graded vector spaces with nontrivial associator $\omega$. The category $\mathcal{C}(\mathcal{D}, \omega)$ is a multi-fusion category as follows. If $M=\oplus_{\alpha \in \mathcal{D}} M_{\alpha}$ and $N=\oplus_{\beta \in \mathcal{D}} N_{\beta}$ are two objects then $M \otimes N:=\bigoplus_{\mathfrak{e}(\alpha)=\mathfrak{s}(\beta)} M_{\alpha} \otimes_{\mathbb{k}} N_{\beta}$ with $\mathcal{D}$-grading determined by

$$
(M \otimes N)_{\alpha}:=\bigoplus_{\alpha_{1} \alpha_{2}=\alpha} M_{\alpha_{1}} \otimes_{\mathbb{k}} N_{\alpha_{2}} .
$$

The associativity constraint on homogeneous elements is given by $\omega$. The unit object is $\mathbb{k} \mathcal{P}$, with grading given by $|P|=\operatorname{id}_{P}, P \in \mathcal{P}$. The unit isomorphisms for this category are canonical. 
The category $\mathcal{C}(\mathcal{D}, \omega)$ is a direct sum of its indecomposable multi-fusion subcategories corresponding to connected components of $\mathcal{D} \rightrightarrows \mathcal{P}$. Therefore $\mathcal{C}(\mathcal{D}, \omega)$ is not an indecomposable multi-fusion category unless $\mathcal{D} \rightrightarrows \mathcal{P}$ is connected.

The irreducible constituents of $\mathbf{1}=\mathbb{k} \mathcal{P}$ are the one-dimensional subspaces $\mathbb{k} P$, $P \in \mathcal{P}$. Therefore, $\mathcal{C}(\mathcal{D}, \omega)$ is a fusion category if and only if $\# \mathcal{P}=1$, that is, if and only if $\mathcal{D}$ is a group. Indeed, the categories $\mathcal{C}_{P, Q}$ of $\mathcal{C}=\mathcal{C}(\mathcal{D}, \omega)$ are the full subcategories whose objects are finite dimensional vector spaces graded by the set $\mathcal{D}(P, Q)$ of arrows going from $P$ to $Q$.

An object $X$ in a multi-fusion category $\mathcal{C}$ will be called invertible if there exists an object $Y$ such that $X \otimes Y$ and $Y \otimes X$ are irreducible summands of the unit object 1 .

Following [ENO] we shall say that a multi-fusion category is pointed if every simple object is invertible. Every pointed multi-fusion category is equivalent to a category of the form $\mathcal{C}(\mathcal{D}, \omega)$ for some finite groupoid $\mathcal{D}$ and 3-cocycle $\omega$.

For all $P \in \mathcal{P}$, the component fusion category $\mathcal{C}_{P, P}$ is exactly the category of $\mathcal{D}(P)$ graded vector spaces with associativity given by the restriction $\widehat{\omega}$ of $\omega$ to $\mathcal{D}(P)$. In particular, every fusion component in $\mathcal{C}$ is pointed group-theoretical.

Suppose that $\mathcal{D} \rightrightarrows \mathcal{P}$ is connected. By [ENO], $\mathcal{M}_{(P)}=\oplus_{Q \in \mathcal{P}} \mathcal{C}_{Q P}$ is an indecomposable module category over $\mathcal{C}$ consisting, in this case, of all objects graded by arrows $\alpha$ with target $\mathfrak{e}(\alpha)=P$, such that $\mathcal{C}_{\mathcal{M}_{(P)}^{*}} \simeq \mathcal{C}(\mathcal{D}(P), \widehat{\omega})^{\mathrm{op}}$.

2.3. The categories $\mathcal{C}(\mathcal{D}, \omega, \mathcal{V}, \psi)$. Let $\mathcal{V} \rightrightarrows \widetilde{\mathcal{P}}$ be a subgroupoid of $\mathcal{D}$ on the base $\widetilde{\mathcal{P}} \subseteq \mathcal{P}$. Let also $\psi: \mathcal{V}_{\mathfrak{e}} \times_{\mathfrak{s}} \mathcal{V} \rightarrow \mathbb{k}^{\times}$be a normalized 2-cochain such that

$$
\left.\omega\right|_{\mathcal{V}_{\mathrm{e}} \times{ }_{\mathfrak{s}} \mathcal{V}_{\mathrm{e}} \times_{\mathfrak{s}} \mathcal{V}}=d \psi
$$

Then the twisted groupoid algebra $\mathbb{k}_{\psi} \mathcal{V}$ is an algebra in $\mathcal{C}(\mathcal{D}, \omega)$.

Remark 2.1. The category $\mathcal{C}(\mathcal{D}, \omega)_{\mathbb{k}_{\psi} \mathcal{V}}$ of right $\mathbb{k}_{\psi} \mathcal{V}$-modules in $\mathcal{C}(\mathcal{D}, \omega)$ is naturally a module category over $\mathcal{C}(\mathcal{D}, \omega)[\mathrm{O} 1]$.

There is a decomposition

$$
\mathcal{C}(\mathcal{D}, \omega)_{\mathbb{k}_{\psi} \mathcal{V}}=\bigoplus_{(P) \in \widetilde{P} / \mathcal{V}}(P) \mathcal{C}(\mathcal{D}, \omega)_{\mathbb{k}_{\psi} \mathcal{V}}
$$

where the module category ${ }_{(P)} \mathcal{C}(\mathcal{D}, \omega)_{\mathbb{k}_{\psi} \mathcal{V}}$ is the full subcategory of $\mathcal{C}(\mathcal{D}, \omega)_{\mathbb{k}_{\psi} \mathcal{V}}$ whose objects are finite dimensional vector spaces graded by arrows with target in the connected component $(P)$ of $\widetilde{P}$ under the action of $\mathcal{V}$.

In addition, the module category ${ }_{(P)} \mathcal{C}(\mathcal{D}, \omega)_{\mathbb{k}_{\psi} \mathcal{V}}$ is indecomposable if and only if the groupoid $\mathcal{V} \rightrightarrows \widetilde{\mathcal{P}}$ is connected.

Denote by $\mathcal{C}(\mathcal{D}, \omega, \mathcal{V}, \psi)$ the tensor category of $\mathbb{k}_{\psi} \mathcal{V}$-bimodules in $\mathcal{C}(\mathcal{D}, \omega)$. The category $\mathcal{C}(\mathcal{D}, \omega, \mathcal{V}, \psi)$ is thus the dual category to $\mathcal{C}(\mathcal{D}, \omega)$ with respect to the module category $\mathcal{C}(\mathcal{D}, \omega)_{\mathbb{k}_{\psi} \mathcal{V}}$. By construction, the categories $\mathcal{C}(\mathcal{D}, \omega, \mathcal{V}, \psi)$ are a multi-fusion generalization of group-theoretical categories.

Remark 2.2. Let $\eta: \mathcal{D}_{\mathfrak{e}} \times{ }_{\mathfrak{s}} \mathcal{D} \rightarrow \mathbb{k}^{\times}, \chi: \mathcal{V} \rightarrow \mathbb{k}^{\times}$be normalized cochains. As in [ENO, Remark 8.39], there are tensor equivalences

$$
\mathcal{C}(\mathcal{D}, \omega, \mathcal{V}, \psi) \simeq \mathcal{C}\left(\mathcal{D}, \omega^{\prime}, \mathcal{V}, \psi^{\prime}\right)
$$

where $\omega^{\prime}=\omega(d \eta), \psi^{\prime}=\left.\psi \eta\right|_{\mathcal{V}}(d \chi)$. 
Recall that a subgroupoid $\mathcal{V} \rightrightarrows \widetilde{\mathcal{P}}$ of $\mathcal{D}$ is called wide if $\widetilde{\mathcal{P}}=\mathcal{P}$.

Lemma 2.2. Suppose $\mathcal{V}$ is a wide subgroupoid. Then $\mathcal{C}(\mathcal{D}, \omega, \mathcal{V}, \psi)$ is a fusion category if and only if $\mathcal{V} \rightrightarrows \mathcal{P}$ is connected.

Proof. Let $\mathcal{P}=\coprod_{(P) \in \mathcal{P} / \mathcal{V}}(P)$ be the decomposition of $\mathcal{P}$ into disjoint equivalence classes under the action of $\mathcal{V}$. Then $\mathbb{k}_{\psi} \mathcal{V}=\oplus_{(P)} \mathbb{k}_{\psi(P)} \mathcal{V}_{(P)}$, where $\mathcal{V}_{(P)}$ is the corresponding connected groupoid on the base $(P)$ and $\psi^{(P)}$ is the restriction of $\psi$ to $\mathcal{V}_{(P)}$. The components $\mathbb{k}_{\psi^{(P)}} \mathcal{V}_{(P)}$ are subobjects of $\mathbb{k}_{\psi} \mathcal{V}$ and it is not difficult to see that $\mathbb{k}_{\psi^{(P)}} \mathcal{V}_{(P)}$ is irreducible as an object of $\mathcal{C}(\mathcal{D}, \omega, \mathcal{V}, \psi)$. This implies the lemma.

Suppose that the 3 -cocycle $\omega$ satisfies

$$
\omega \mid \mathcal{V}_{\mathfrak{e}} \times_{\mathfrak{s}} \mathcal{V}_{\mathfrak{e}} \times_{\mathfrak{s}} \mathcal{V}=1
$$

Objects in $\mathcal{C}(\mathcal{D}, \omega, \mathcal{V}):=\mathcal{C}(\mathcal{D}, \omega, \mathcal{V}, 1)$ can be described explicitly as follows: these are vector spaces graded by arrows in $\mathcal{D}$ with source and target in $\widetilde{\mathcal{P}}$,

$$
M=\oplus_{\left.\alpha \in \mathcal{D}\right|_{\tilde{\mathcal{P}}}} M_{\alpha},
$$

together with actions of $\mathcal{V}$ by linear isomorphisms:

$$
g \rightarrow: M_{\alpha} \rightarrow M_{g \alpha}, \quad \leftarrow h: M_{\alpha} \rightarrow M_{\alpha h}
$$

$g, h \in \mathcal{V}, \alpha \in \mathcal{D}$, such that $\mathfrak{e}(g)=\mathfrak{s}(\alpha), \mathfrak{s}(h)=\mathfrak{e}(\alpha)$.

Letting $|m| \in \mathcal{D}$ denote the homogeneity degree of an homogeneous element $m \in$ $M$, the following relations are satisfied:

$$
\begin{gathered}
\mid m\llcorner h|=| m|h, \quad| g \rightarrow m|=g| m \mid, \\
g h \rightarrow m=\omega(g, h,|m|) g \rightarrow(h \rightarrow m), \\
\operatorname{id}_{p} \rightarrow m= \begin{cases}m & \text { if } p=\mathfrak{s}(|m|) \in \widetilde{\mathcal{P}}, \\
0 & \text { otherwise, }\end{cases} \\
(m\llcorner g) \leftarrow h=\omega(|m|, g, h) m\llcorner g h, \\
m \leftarrow \operatorname{id}_{p}= \begin{cases}m & \text { if } p=\mathfrak{e}(|m|) \in \widetilde{\mathcal{P}}, \\
0 & \text { otherwise },\end{cases} \\
(g \rightarrow m) \leftarrow h=\omega(g,|m|, h) g \rightarrow(m \leftarrow h),
\end{gathered}
$$

for any homogeneous element $m \in M, g, h \in \mathcal{V}$, such that the elements $g,|m|, h$ are composable in $\mathcal{D}$.

The tensor structure on the category $\mathcal{C}(\mathcal{D}, \omega, \mathcal{V})$ can be described as follows. If $M, N \in \mathcal{C}(\mathcal{D}, \omega, \mathcal{V})$ denote by $V(M, N)$ the (graded) subspace of $M \otimes N$ spanned by

$$
(m<g) \otimes n-\omega(|m|, g,|n|) m \otimes(g \rightarrow n),
$$

where $m \in M, n \in N$, are homogeneous elements such that $|m|, g,|n|$ are composable in $\mathcal{D}$.

Tensor product $\bar{\otimes}$ on $\mathcal{C}(\mathcal{D}, \omega, \mathcal{V})$ is defined by $M \bar{\otimes} N=(M \otimes N) / V(M, N)$, with $\mathcal{D}$-grading inherited from $M \otimes N$. The left and right actions of $\mathbb{k} \mathcal{V}$ on $M \bar{\otimes} N$ are as follows. The class of $m \otimes n$ will be denoted by $m \bar{\otimes} n$. Assume that $m \in M, n \in N$ 
are homogeneous elements such that $\mathfrak{e}(|m|)=\mathfrak{s}(|n|)$. Let $g, h \in \mathcal{V}$ such that $\mathfrak{e}(g)=$ $\mathfrak{s}(|m|), \mathfrak{e}(|n|)=\mathfrak{s}(h)$, then

$$
\begin{aligned}
& g \rightarrow(m \bar{\otimes} n)=\omega^{-1}(g,|m|,|n|)(g \rightarrow m) \bar{\otimes} n, \\
& (m \bar{\otimes} n) \leftarrow h=\omega(|m|,|n|, h) m \bar{\otimes}(n \leftarrow h) .
\end{aligned}
$$

The unit object is $\mathbb{k} \mathcal{V}$.

Example 2.3. According to the above description, for all $P \in \mathcal{P}$, there is a natural identification

$$
\mathcal{C}(\mathcal{D}, \omega, \mathcal{V}(P))=\mathcal{C}(\mathcal{D}(P), \widehat{\omega}, \mathcal{V}(P))
$$

hence $\mathcal{C}(\mathcal{D}, \omega, \mathcal{V}(P))$ is a fusion group-theoretical category.

Example 2.4. Let $P \in \mathcal{P}$, and consider the subgroupoid

$$
\left\{\operatorname{id}_{P}\right\}=\mathcal{V}_{(P)} \rightrightarrows \widetilde{\mathcal{P}}=\{P\} \text {. }
$$

In this case, the category $\mathcal{C}(\mathcal{D}, \omega)_{\mathbb{k}} \mathcal{V}_{(P)}$ of right $\mathbb{k} \mathcal{V}_{(P)}$-modules in $\mathcal{C}(\mathcal{D}, \omega)$ is the full tensor subcategory whose objects are graded by arrows $\alpha$ with $\mathfrak{e}(\alpha)=P$.

This coincides with the module category $\mathcal{M}_{(P)}=\oplus_{Q \in \mathcal{P}} \mathcal{C}_{Q P}$ [ENO, 2.4].

Suppose that the groupoid $\mathcal{D} \rightrightarrows \mathcal{P}$ is connected. Let $D=\mathcal{D}(P)$, and $\widehat{\omega}$ the 3 cocycle on $D$ obtained by restriction. As remarked before, by the results in the proof of Proposition 2.17 in [ENO, 5.5], $\mathcal{M}_{(P)}$ is an indecomposable module category and there are isomorphisms

$$
\mathcal{C}_{\mathcal{M}_{(P)}}^{*} \simeq \mathcal{C}\left(\mathcal{D}, \omega, \mathcal{V}_{(P)}\right) \simeq \mathcal{C}(D, \widehat{\omega})^{\mathrm{op}}
$$

The last isomorphism can also be seen as a consequence of the description of the category $\mathcal{C}\left(\mathcal{D}, \omega, \mathcal{V}_{(P)}\right)$ given above.

In particular, the category $\mathcal{C}\left(\mathcal{D}, \omega, \mathcal{V}_{(P)}\right)$ is a fusion group-theoretical category, even though the groupoid $\mathcal{V}_{(P)}$ may not be a wide subgroupoid, that is, we may have $\widetilde{\mathcal{P}} \neq \mathcal{P}$.

2.4. The connected case. From now on we shall assume that $\mathcal{V} \rightrightarrows \widetilde{\mathcal{P}}$ is a wide subgroupoid (so $\widetilde{\mathcal{P}}=\mathcal{P}$ ) which is connected. Thus $\mathcal{D} \rightrightarrows \mathcal{P}$ is also connected. We shall also fix a 3 -cocycle $\omega$ on $\mathcal{D}$ such that

$$
\omega \mid \mathcal{V}_{\mathrm{e}} \times_{\mathrm{s}} \mathcal{V}_{\mathrm{e}} \times_{\mathrm{s}} \mathcal{V}=1
$$

The connectedness assumption on $\mathcal{V}$ implies that the unit object $\mathbb{k} \mathcal{V}$ in the category $\mathcal{C}(\mathcal{D}, \omega, \mathcal{V})$ is irreducible. Therefore, $\mathcal{C}(\mathcal{D}, \omega, \mathcal{V})$ is in this case a fusion category. In what follows we shall give an explicit equivalence that shows that $\mathcal{C}(\mathcal{D}, \omega, \mathcal{V})$ is indeed a group-theoretical category.

Fix an element $O \in \mathcal{P}$. Since $\mathcal{V}$ is connected, for any $P \in \mathcal{P}$, we can choose an element $\tau_{P} \in \mathcal{V}(O, P)$. There is no harm to assume $\tau_{O}=\mathrm{id}{ }_{O}$.

Set $V=\mathcal{V}(O), D=\mathcal{D}(O)$. For all $P, Q \in \mathcal{P}$ we have

$$
\mathcal{D}(P, Q)=\tau_{P}^{-1} D \tau_{Q}, \quad \mathcal{V}(P, Q)=\tau_{P}^{-1} V \tau_{Q} .
$$

Let $\widehat{\omega}$ be the restriction of $\omega$ to $D$; so $\widehat{\omega}$ gives a well-defined 3 -cocycle on $D$. This restriction map determines a group isomorphism

$$
H^{3}\left(\mathcal{D}, \mathbb{k}^{\times}\right) \rightarrow H^{3}\left(D, \mathbb{k}^{\times}\right), \quad \omega \mapsto \widehat{\omega} .
$$


See for instance $[\mathrm{AM}]$.

Let $\widetilde{\omega} \in Z^{3}\left(\mathcal{D}, \mathbb{k}^{\times}\right)$be the 3 -cocycle defined by the formula

$$
\widetilde{\omega}(g, h, t)=\widehat{\omega}\left(\tau_{P} g \tau_{Q}^{-1}, \tau_{Q} h \tau_{R}^{-1}, \tau_{R} t \tau_{S}^{-1}\right),
$$

for $g \in \mathcal{D}(P, Q), h \in \mathcal{D}(Q, R), t \in \mathcal{D}(R, S)$.

Note that, since $\tau_{P} \in \mathcal{V}$, for all $P \in \mathcal{P}$, then we also have $\widetilde{\omega} \mid \mathcal{V}_{\mathfrak{e}} \times{ }_{\mathfrak{s}} \mathcal{V}_{\mathfrak{e}} \times \mathfrak{s} \mathcal{V}=1$.

By construction, $\left.\widetilde{\omega}\right|_{D}=\widehat{\omega}$. Therefore $\widetilde{\omega}$ is cohomologous to $\omega$, say

$$
\widetilde{\omega}=\omega(d \psi),
$$

for some normalized 2-cochain $\psi: \mathcal{D}_{\mathfrak{e}} \times{ }_{\mathfrak{s}} \mathcal{D} \rightarrow \mathbb{k}^{\times}$.

In particular, the tensor categories $\mathcal{C}(\mathcal{D}, \omega)$ and $\mathcal{C}(\mathcal{D}, \widetilde{\omega})$ are equivalent.

Moreover, since both restrictions $\left.\omega\right|_{\mathcal{V}}$ and $\left.\widetilde{\omega}\right|_{\mathcal{V}}$ are trivial, relation (2.12) implies that the restriction $\psi: \mathcal{V}_{\mathfrak{e}} \times_{\mathfrak{s}} \mathcal{V} \rightarrow \mathbb{k}^{\times}$is a 2-cocycle, and by Remark 2.2 there is a tensor equivalence

$$
\mathcal{C}(\mathcal{D}, \omega, \mathcal{V}) \simeq \mathcal{C}(\mathcal{D}, \widetilde{\omega}, \mathcal{V}, \psi)
$$

Let $\widehat{\psi}=\left.\psi\right|_{V}: V \times V \rightarrow \mathbb{k}^{\times}$be the corresponding 2-cocycle on $V$, and let also $\widetilde{\psi}: \mathcal{V}_{\mathfrak{e}} \times_{\mathfrak{s}} \mathcal{V} \rightarrow \mathbb{k}^{\times}$be the 2-cocycle defined by the formula

$$
\widetilde{\psi}(g, h):=\widehat{\psi}\left(\tau_{P} g \tau_{Q}^{-1}, \tau_{Q} h \tau_{R}^{-1}\right),
$$

for all $g \in \mathcal{V}(P, Q), h \in \mathcal{V}(Q, R)$. Repeating the argument used above for $\omega$, the results in $[\mathrm{AM}]$ imply that $\psi$ and $\widetilde{\psi}$ differ by a 2 -coboundary, that is,

$$
\widetilde{\psi}=\psi(d \chi),
$$

on the connected subgroupoid $\mathcal{V}$, where $\chi: \mathcal{V} \rightarrow \mathbb{k}^{\times}$is a normalized 1-cochain.

Combining this fact with the equivalence (2.13) and Remark 2.2 we get tensor equivalences

$$
\mathcal{C}(\mathcal{D}, \omega, \mathcal{V}) \simeq \mathcal{C}(\mathcal{D}, \widetilde{\omega}, \mathcal{V}, \psi) \simeq \mathcal{C}(\mathcal{D}, \widetilde{\omega}, \mathcal{V}, \widetilde{\psi})
$$

Let $M \in \mathcal{C}(\mathcal{D}, \widetilde{\omega}, \mathcal{V}, \widetilde{\psi})$. Let us define $F(M) \in \mathcal{C}(D, \widehat{\omega}, V, \widehat{\psi})$ by

$$
F(M)=\bigoplus_{z \in D} M_{z}
$$

This gives us a well-defined functor $F: \mathcal{C}(\mathcal{D}, \widetilde{\omega}, \mathcal{V}, \widetilde{\psi}) \rightarrow \mathcal{C}(D, \widehat{\omega}, V, \widehat{\psi})$.

Note that, for $M \in \mathcal{C}(\mathcal{D}, \widetilde{\omega}, \mathcal{V}, \widetilde{\psi})$,

$$
M=\bigoplus_{P, Q \in \mathcal{P}} \tau_{P}^{-1} \rightarrow F(M)<\tau_{Q} .
$$

Proposition 2.5. The functor $F$ is an equivalence of tensor categories

$$
F: \mathcal{C}(\mathcal{D}, \widetilde{\omega}, \mathcal{V}, \widetilde{\psi}) \stackrel{\simeq}{\rightarrow}(D, \widehat{\omega}, V, \widehat{\psi}) .
$$


Proof. Define a functor $G: \mathcal{C}(D, \widehat{\omega}, V, \widehat{\psi}) \rightarrow \mathcal{C}(\mathcal{D}, \widetilde{\omega}, \mathcal{V}, \widetilde{\psi})$ by the formula

$$
G(W)=\bigoplus_{P, Q \in \mathcal{P}} \bigoplus_{z \in D} W_{(P, z, Q)} \simeq \mathbb{k} \mathcal{P} \otimes_{\mathbb{k}} W \otimes_{\mathbb{k}} \mathbb{k} \mathcal{P},
$$

for all $P, Q \in \mathcal{P}, z \in D$, where

$$
W_{(P, z, Q)}=\mathbb{k} \tau_{P}^{-1} \otimes_{\mathbb{k}} W_{z} \otimes_{\mathbb{k}} \mathbb{k} \tau_{Q},
$$

is an object in the category $\mathcal{C}(\mathcal{D}, \widetilde{\omega}, \mathcal{V}, \widetilde{\psi})$ as follows: all elements in $W_{(P, z, Q)}$ are homogeneous of degree $\tau_{P}^{-1} z \tau_{Q}$. If $v \in W_{z}$ and $g \in \mathcal{V}(R, P), h \in \mathcal{V}(Q, S)$ then the left and right actions are defined by

$$
\begin{aligned}
& g \rightarrow \tau_{P}^{-1} \otimes w \otimes \tau_{Q}=\tau_{R}^{-1} \otimes\left(\tau_{R} g \tau_{P}^{-1}\right) \cdot w \otimes \tau_{Q} \in W_{(R, z, Q)}, \\
& \tau_{P}^{-1} \otimes w \otimes \tau_{Q} \leftarrow h=\tau_{P}^{-1} \otimes w \cdot\left(\tau_{Q} h \tau_{S}^{-1}\right) \otimes \tau_{S} \in W_{(P, z, S)} .
\end{aligned}
$$

Formulas (2.11) and (2.14) imply that $G$ is well defined.

We claim that the functors $F$ and $G$ are inverse tensor equivalences.

Because $\left.\widetilde{\omega}\right|_{D}=\widehat{\omega}$ and $\left.\widetilde{\psi}\right|_{V}=\widehat{\psi}$, we have $F(G(W)) \simeq W$, for all $W \in \mathcal{C}(D, \widehat{\omega}, V, \widehat{\psi})$.

The proof of the proposition will be finished once we have established the following claim. Its proof uses connectedness of the groupoid $\mathcal{V} \rightrightarrows \mathcal{P}$.

Claim 2.1. The map

$$
f:\left(\mathbb{k} \tau_{P}^{-1} \otimes_{\mathbb{k}} U \otimes_{\mathbb{k}} \mathbb{k} \tau_{Q}\right) \otimes_{\mathbb{k}}\left(\mathbb{k} \tau_{Q}^{-1} \otimes_{\mathbb{k}} W \otimes_{\mathbb{k}} \mathbb{k} \tau_{S}\right) \rightarrow \mathbb{k} \tau_{P}^{-1} \otimes_{\mathbb{k}}(U \bar{\otimes} W) \otimes_{\mathbb{k}} \mathbb{k} \tau_{S},
$$

determined by

$$
\tau_{P}^{-1} \otimes_{\mathbb{k}} u \otimes_{\mathbb{k}} \tau_{Q} \otimes_{\mathbb{k}} \tau_{Q}^{-1} \otimes_{\mathbb{k}} w \otimes_{\mathbb{k}} \tau_{S} \mapsto \tau_{P}^{-1} \otimes_{\mathbb{k}}(u \bar{\otimes} w) \otimes_{\mathbb{k}} \tau_{S},
$$

induces a natural isomorphism $\zeta_{U, W}: G(U) \bar{\otimes} G(W) \rightarrow G(U \bar{\otimes} W)$. This isomorphism endows $G$ with a tensor functor structure.

Recall that $\otimes$ means tensor product in the category of $\mathcal{D}$-graded spaces.

Proof of the Claim. First note that $f$ defines a map from the space

$$
\left(\mathbb{k} \mathcal{P} \otimes_{\mathbb{k}} U \otimes_{\mathbb{k}} \mathbb{k} \mathcal{P}\right) \otimes\left(\mathbb{k} \mathcal{P} \otimes_{\mathbb{k}} W \otimes_{\mathbb{k}} \mathbb{k} \mathcal{P}\right)=\bigoplus_{P, Q, S} \mathbb{k} \tau_{P}^{-1} \otimes_{\mathbb{k}} U \otimes_{\mathbb{k}} \mathbb{k} \tau_{Q} \otimes_{\mathbb{k}} \mathbb{k} \tau_{Q}^{-1} \otimes_{\mathbb{k}} W \otimes_{\mathbb{k}} \mathbb{k} \tau_{S}
$$

to the space

$$
G(U \bar{\otimes} W)=\bigoplus_{P, S} \mathbb{k} \tau_{P}^{-1} \otimes_{\mathbb{k}}(U \bar{\otimes} W) \otimes_{\mathbb{k}} \mathbb{k} \tau_{S} .
$$

Let now $g \in \mathcal{V}$ with $\mathfrak{s}(g)=Q, \mathfrak{e}(g)=Q^{\prime}$. Then we have

$$
\begin{aligned}
& f\left(\left(\tau_{P}^{-1} \otimes_{\mathbb{k}} u \otimes_{\mathbb{k}} \tau_{Q}\right)<g \otimes_{\mathbb{k}} \tau_{Q^{\prime}}^{-1} \otimes_{\mathbb{k}} w \otimes_{\mathbb{k}} \tau_{S}\right) \\
& =f\left(\tau_{P}^{-1} \otimes_{\mathbb{k}} u \cdot\left(\tau_{Q} g \tau_{Q^{\prime}}^{-1}\right) \otimes_{\mathbb{k}} \tau_{Q^{\prime}} \otimes_{\mathbb{k}} \tau_{Q^{\prime}}^{-1} \otimes_{\mathbb{k}} w \otimes_{\mathbb{k}} \tau_{S}\right) \\
& =\tau_{P}^{-1} \otimes_{\mathbb{k}} \bar{u}\left(\tau_{Q} g \tau_{Q^{\prime}}^{-1}\right) \otimes_{\mathbb{k}} w \otimes_{\mathbb{k}} \tau_{S} \\
& =\widehat{\omega}\left(|u|, \tau_{Q} g \tau_{Q^{\prime}}^{-1},|w|\right) \tau_{P}^{-1} \otimes_{\mathbb{k}} \overline{u \otimes_{\mathbb{k}}\left(\tau_{Q} g \tau_{Q^{\prime}}^{-1}\right) \cdot w} \otimes_{\mathbb{k}} \tau_{S} \\
& =\widetilde{\omega}\left(\tau_{P}|u| \tau_{Q}^{-1}, g, \tau_{Q^{\prime}}|w| \tau_{S}^{-1}\right) f\left(\tau_{P}^{-1} \otimes_{\mathbb{k}} u \otimes_{\mathbb{k}} \tau_{Q} \otimes_{\mathbb{k}} g \rightarrow\left(\tau_{Q^{\prime}}^{-1} \otimes_{\mathbb{k}} w \otimes_{\mathbb{k}} \tau_{S}\right)\right) .
\end{aligned}
$$

Hence $f$ induces a map $\zeta_{U, W}: G(U) \bar{\otimes} G(W) \rightarrow G(U \bar{\otimes} W)$. Using (2.10) and (2.11) we see that $\zeta_{U, W}$ is a map in $\mathcal{C}(\mathcal{D}, \widetilde{\omega}, \mathcal{V}, \widetilde{\psi})$. 
Let $\bar{f}: \mathbb{k} \mathcal{P} \otimes_{\mathbb{k}} U \otimes_{\mathbb{k}} W \otimes_{\mathbb{k}} \mathbb{k} \mathcal{P} \rightarrow G(U) \bar{\otimes} G(W)$ be determined by

$$
\bar{f}\left(\tau_{P}^{-1} \otimes_{\mathbb{k}} u \otimes_{\mathbb{k}} w \otimes_{\mathbb{k}} \tau_{S}\right)=\tau_{P}^{-1} \otimes_{\mathbb{k}} u \otimes_{\mathbb{k}} \tau_{O} \otimes \tau_{O} \otimes_{\mathbb{k}} w \otimes_{\mathbb{k}} \tau_{S} .
$$

Recall that $\tau_{O}=\mathrm{id} \mathrm{O}_{O}$. A similar computation to the one done before shows that $\bar{f}$ induces a map $\xi_{U, W}: G(U \bar{\otimes} W) \rightarrow G(U) \bar{\otimes} G(W)$. Clearly $\zeta_{U, V} \xi_{U, V}=\mathrm{id}$. On the other hand, for all $P, Q, S \in \mathcal{P}$, we have

$$
\begin{aligned}
\tau_{P}^{-1} \otimes_{\mathbb{k}} u \otimes_{\mathbb{k}} \tau_{Q} \bar{\otimes} \tau_{Q}^{-1} \otimes_{\mathbb{k}} w \otimes_{\mathbb{k}} \tau_{S} \\
=\left(\tau_{P}^{-1} \otimes_{\mathbb{k}} u \otimes_{\mathbb{k}} \tau_{O}\right) \leftarrow \tau_{Q} \bar{\otimes} \tau_{Q}^{-1} \rightarrow\left(\tau_{O} \otimes_{\mathbb{k}} w \otimes_{\mathbb{k}} \tau_{S}\right) \\
=\tau_{P}^{-1} \otimes_{\mathbb{k}} u \otimes_{\mathbb{k}} \tau_{O} \bar{\otimes} \tau_{O} \otimes_{\mathbb{k}} w \otimes_{\mathbb{k}} \tau_{S},
\end{aligned}
$$

because $\widetilde{\omega}$ and $\widetilde{\psi}$ are trivial whenever any one of their arguments is a $\tau_{Q}$. This implies that $\xi_{U, V} \zeta_{U, V}=\mathrm{id}$. Therefore $\xi_{U, V}$ is inverse to $\zeta_{U, W}$, whence $\zeta$ is an isomorphism.

The definition of $\widetilde{\omega}$ also implies that $\zeta$ is compatible with the associativity and unit constraints. This finishes the proof of the claim.

Corollary 2.6. There is a tensor equivalence $\mathcal{C}(\mathcal{D}, \omega, \mathcal{V}) \simeq \mathcal{C}(D, \widehat{\omega}, V, \widehat{\psi})$. In particular, the category $\mathcal{C}(\mathcal{D}, \omega, \mathcal{V})$ is group-theoretical.

Proof. It follows from (2.15) and Proposition 2.5.

\section{Vacant double groupoids}

According to the definition given by Ehresmann [E], a double groupoid is a groupoid object in the category of groupoids. A double groupoid can be represented in the form of four related groupoids

$$
\begin{array}{rlll}
\mathcal{T}: & \mathcal{B} & \rightrightarrows & \mathcal{H} \\
& \downarrow \\
& \mathcal{V} & & \downarrow \\
\mathcal{P}
\end{array}
$$

subject to a set of axioms.

This structure admits a pictorial description as 'boxes' that can be composed in two directions: horizontal and vertical. Throughout we shall work with the conventions and notations from [AN1, Section 2]. An element $A \in \mathcal{B}$ is depicted as a box

$$
A=l \square_{b}^{t} r,
$$

where $t=t(A), b=b(A) \in \mathcal{H}$ are respectively the source and target of $A$ with respect to vertical composition, and similarly for $l=l(A), r=r(A) \in \mathcal{V}$ with respect to horizontal composition.

Horizontal and vertical composition of boxes will be written from left to right and from top to bottom, respectively. The notation $A B$ (respectively, $\underset{B}{A}$ ) will indicate the horizontal (respectively, vertical) composition; this notation will always implicitly assume that $A$ and $B$ are composable in the appropriate sense. 
A double groupoid $\mathcal{T}$ is called vacant if for any $g \in \mathcal{V}, x \in \mathcal{H}$ such that the target of $x$ coincides with the source of $g$, there is exactly one box $X \in \mathcal{B}$ such that $X=\square g$.

In particular, in a vacant double groupoid, every box is determined by (any) pair of adjacent edges.

3.1. Matched pairs of groupoids. Let $\mathfrak{s}, \mathfrak{e}: \mathcal{G} \rightrightarrows \mathcal{P}$ be a groupoid with source and target maps $\mathfrak{s}$ and $\mathfrak{e}$. A left action of $\mathcal{G}$ on a map $p: \mathcal{E} \rightarrow \mathcal{P}$ is a map $\triangleright: \mathcal{G} \mathfrak{e} \times{ }_{p} \mathcal{E} \rightarrow \mathcal{E}$ such that, for all composable $g, h \in \mathcal{G}, x \in \mathcal{E}$,

$$
p(g \triangleright x)=\mathfrak{s}(g), \quad g \triangleright(h \triangleright x)=g h \triangleright x, \quad \operatorname{id}_{p(x)} \triangleright x=x .
$$

Similarly, a right action of $\mathcal{G}$ on $\mathcal{E}$ is a map $\triangleleft: \mathcal{E}_{p} \times_{\mathfrak{s}} \mathcal{G} \rightarrow \mathcal{E}$ such that

$$
p(x \triangleleft g)=\mathfrak{e}(g), \quad(x \triangleright g) \triangleright h=x \triangleleft g h, \quad x \triangleleft \mathrm{id}_{p(x)}=x,
$$

for all composable $g, h \in \mathcal{G}, x \in \mathcal{E}$.

A matched pair of groupoids is a collection $(\mathcal{H}, \mathcal{V}, \triangleright, \triangleleft)$, where $t, b: \mathcal{V} \rightrightarrows \mathcal{P}$ and $l, r: \mathcal{H} \rightrightarrows \mathcal{P}$ are two groupoids on the same base $\mathcal{P}, \triangleright: \mathcal{H}_{r} \times_{t} \mathcal{V} \rightarrow \mathcal{V}$ is a left action of $\mathcal{H}$ on $(\mathcal{V}, b)$ and $\triangleleft: \mathcal{H}_{r} \times_{t} \mathcal{V} \rightarrow \mathcal{H}$ is a right action of $\mathcal{V}$ on $(\mathcal{H}, l)$ satisfying the following conditions:

$$
r(x \triangleright g)=t(x \triangleleft g), \quad x \triangleright g h=(x \triangleright g)((x \triangleleft g) \triangleright h), \quad x y \triangleleft g=(x \triangleleft(y \triangleright g))(y \triangleleft g),
$$

for composable elements $x, y \in \mathcal{H}$ and $g, h \in \mathcal{V}$.

3.2. Exact factorizations. Let $(\mathcal{H}, \mathcal{V}, \triangleleft, \triangleright)$ be a matched pair of groupoids. There is an associated diagonal groupoid $\mathcal{D} \rightrightarrows \mathcal{P}$ with arrows set $\mathcal{D}=\mathcal{V}_{\mathfrak{e}} \times_{\mathfrak{s}} \mathcal{H}$, and source, target, composition and identity given by

$$
\begin{aligned}
& \mathfrak{s}(g, x)=\mathfrak{s}(g), \quad \mathfrak{e}(g, x)=\mathfrak{e}(x), \\
& (g, x)(h, y)=(g(x \triangleright h),(x \triangleleft h) y), \quad \operatorname{id}_{P}=\left(\operatorname{id}_{P}, \operatorname{id}_{P}\right),
\end{aligned}
$$

$g, h \in \mathcal{V}, x, y \in \mathcal{H}, P \in \mathcal{P}$. The diagonal groupoid $\mathcal{D}$ admits an exact factorization as the product of its wide subgroupoids $\mathcal{V}$ and $\mathcal{H}$. In what follows we shall use the notation $\mathcal{D}=\mathcal{V} \bowtie \mathcal{H}$.

3.3. Characterization. Out of each matched pair of groupoids $(\mathcal{H}, \mathcal{V})$ one can build a vacant double groupoid with boxes $\mathcal{B}:=\mathcal{H}_{r} \times_{t} \mathcal{V}$. The element $X=(x, g) \in \mathcal{H}_{r} \times_{t} \mathcal{V}$ can be represented by $X=x \triangleright g \underset{x \triangleleft g}{{ }_{x}} g$.

More precisely, the following result due to Mackenzie says that the three notions discussed in this section are indeed equivalent ones.

Proposition 3.1. [M, Theorems 2.10 and 2.15] The following notions are equivalent.

(1) Matched pairs of groupoids.

(2) Groupoids with an exact factorization.

(3) Vacant double groupoids.

We refer the reader to [AN1] for more details on these equivalences. 


\section{Weak Hopf algebras arising from vacant double groupoids}

Throughout this section we shall consider a finite vacant double groupoid $\mathcal{T}$. We recall the weak Hopf algebra introduced in [AN1] arising from $\mathcal{T}$.

Let us first recall the definition of $\operatorname{Opext}(\mathcal{V}, \mathcal{H})$. As a set $\operatorname{Opext}(\mathcal{V}, \mathcal{H})$ consists of equivalence classes of pairs $(\sigma, \tau)$, where $\sigma$ is a normalized 2-cocycle for the vertical groupoid $\mathcal{B} \rightrightarrows \mathcal{V}$ and $\tau$ a normalized 2-cocycle for the horizontal groupoid $\mathcal{B} \rightrightarrows \mathcal{H}$, such that

$$
\sigma(A B, C D) \tau\left(\begin{array}{ll}
A & B \\
C^{\prime} & D
\end{array}\right)=\tau(A, B) \tau(C, D) \sigma(A, C) \sigma(B, D),
$$

for all appropriately composable $A, B, C, D \in \mathcal{B}$.

The set $\operatorname{Opext}(\mathcal{V}, \mathcal{H})$ has a group structure and it fits into the following generalization of the so called Kac exact sequence, see [AN1], [AM]:

$$
\begin{aligned}
0 & \longrightarrow H^{1}\left(\mathcal{D}, \mathbb{k}^{\times}\right) \stackrel{\text { res }}{\longrightarrow} H^{1}\left(\mathcal{H}, \mathbb{k}^{\times}\right) \oplus H^{1}\left(\mathcal{V}, \mathbb{k}^{\times}\right) \longrightarrow \operatorname{Aut}(\mathbb{k} \mathcal{T}) \\
& \longrightarrow H^{2}\left(\mathcal{D}, \mathbb{k}^{\times}\right) \stackrel{\text { res }}{\longrightarrow} H^{2}\left(\mathcal{H}, \mathbb{k}^{\times}\right) \oplus H^{2}\left(\mathcal{V}, \mathbb{k}^{\times}\right) \longrightarrow \operatorname{Opext}(\mathcal{V}, \mathcal{H}) \\
& \longrightarrow H^{3}\left(\mathcal{D}, \mathbb{k}^{\times}\right) \stackrel{\text { res }}{\longrightarrow} H^{3}\left(\mathcal{H}, \mathbb{k}^{\times}\right) \oplus H^{3}\left(\mathcal{V}, \mathbb{k}^{\times}\right) \longrightarrow \ldots,
\end{aligned}
$$

where res denotes the restriction maps.

Remark 4.3. Let $(\sigma, \tau)$ be a representative of a class in Opext $(\mathbb{k} \mathcal{T})$. The map $\operatorname{Opext}(\mathbb{k} \mathcal{T}) \rightarrow H^{3}\left(\mathcal{D}, \mathbb{k}^{\times}\right)$in the above sequence takes the class of $(\sigma, \tau)$ to the class of the 3-cocycle $\omega=\omega(\sigma, \tau)$ defined by

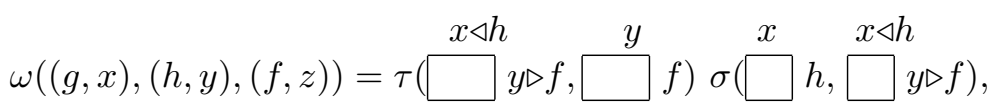

for all composable $(g, x),(h, y),(f, z) \in \mathcal{V} \bowtie \mathcal{H}$. Compare with [Sch, 6.4].

Proof. We only sketch the proof. The Kac exact sequence (4.2) comes from a short exact sequence of double complexes

$$
0 \rightarrow \mathcal{A}^{\bullet, \bullet}\left(\mathbb{k}^{\times}\right) \rightarrow \mathcal{B}^{\bullet \bullet \bullet}\left(\mathbb{k}^{\times}\right) \rightarrow \mathcal{E}^{\bullet \bullet \bullet}\left(\mathbb{k}^{\times}\right) \rightarrow 0,
$$

see $[\mathrm{AN} 1],[\mathrm{AM}]$. The associated total complex of the double complex $\mathcal{B}^{\bullet \bullet},\left(\mathbb{k}^{\times}\right)$is a "non-standard" projective resolution of the trivial $\mathbb{k} \mathcal{D}$-module, hence there are isomorphisms $\zeta_{n}: H^{n}\left(\operatorname{Tot} \mathcal{B}\left(\mathbb{k}^{\times}\right)\right) \stackrel{\simeq}{\rightarrow} H^{n}\left(\mathcal{D}, \mathbb{k}^{\times}\right)$for all $n \geq 0$, that can be computed explicitly. The map $\operatorname{Opext}(\mathcal{V}, \mathcal{H}) \rightarrow H^{3}\left(\mathcal{D}, \mathbb{k}^{\times}\right)$is obtained by composing the map $\operatorname{Opext}(\mathcal{V}, \mathcal{H})=H^{2}\left(\operatorname{Tot} \mathcal{A}^{\bullet \bullet \bullet}\left(\mathbb{k}^{\times}\right)\right) \rightarrow H^{3}\left(\operatorname{Tot} \mathcal{B}^{\bullet \bullet \bullet}\left(\mathbb{k}^{\times}\right)\right)$associated to the inclusion $\mathcal{A}^{\bullet \bullet \bullet}\left(\mathbb{k}^{\times}\right) \rightarrow \mathcal{B}^{\bullet \bullet \bullet}\left(\mathbb{k}^{\times}\right)$with $\zeta_{3}$.

We note for future use the following properties of the Kac 3-cocycle $\omega=\omega(\sigma, \tau)$ :

(4.5) $\left.\omega\right|_{\mathcal{V} \times \mathcal{D} \times \mathcal{D}}=1$,

(4.6) $\omega((g, x),(h, y),(f, z))=\omega\left(\left(\operatorname{id}_{\mathfrak{s}(x)}, x\right),(h, y),\left(f, \mathrm{id}_{\mathfrak{e}(f)}\right)\right)$,

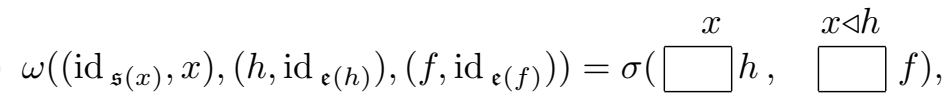


for all appropriate choice of $(g, x),(h, y),(f, z) \in \mathcal{D}$.

Formulas (4.6) and (4.7) are a consequence of (4.4), and (4.5) follows from Remark 3.7 in [AN1].

We now fix a representative $(\sigma, \tau)$ of a class in $\operatorname{Opext}(\mathcal{V}, \mathcal{H})$. We denote by $\mathbb{k}_{\tau}^{\sigma} \mathcal{T}$ the vector space spanned by the boxes of $\mathcal{T}$ endowed with the $\sigma$-twisted groupoid algebra of the vertical composition groupoid and the dual $\tau$-twisted groupoid algebra of the horizontal composition groupoid.

That is, the comultiplication and multiplication in $\mathbb{k}_{\tau}^{\sigma} \mathcal{T}$ are determined by:

$$
\Delta(A)=\sum_{A=B C} \tau(B, C) \quad B \otimes C ; \quad A . B=\left\{\begin{array}{l}
\sigma(A, B) \frac{A}{B}, \quad \text { if } \frac{A}{B}, \\
0, \quad \text { otherwise. }
\end{array}\right.
$$

Consider, for any $P \in \mathcal{P}$, the elements

$$
{ }_{P} \mathbf{1}=\sum_{x \in \mathcal{H}, l(x)=P} \text { id } x, \quad \mathbf{1}_{P}=\sum_{x \in \mathcal{H}, r(x)=P} \text { id } x .
$$

Theorem 4.1. ([AN1, Theorem 3.8]) $\mathbb{k}_{\tau}^{\sigma} \mathcal{T}$ is a semisimple weak Hopf algebra with antipode given by

$$
\mathcal{S}(A)=\tau\left(A, A^{h}\right)^{-1} \sigma\left(A^{-1}, A^{h}\right)^{-1} A^{-1} .
$$

The source and target subalgebras are the subspaces spanned by $\left(\mathbf{1}_{P}\right)_{P \in \mathcal{P}}$ and $\left({ }_{P} \mathbf{1}\right)_{P \in \mathcal{P}}$, respectively; so they are commutative of dimension $|\mathcal{P}|$.

The weak Hopf algebra structure on $\mathbb{k}_{\tau}^{\sigma} \mathcal{T}$ gives rise to a multi-fusion category structure on the category $\operatorname{Rep}\left(\mathbb{k}_{\tau}^{\sigma} \mathcal{T}\right)$ of its finite-dimensional representations. This category is a fusion category if and only if the groupoid $\mathcal{V} \rightrightarrows \mathcal{P}$ is connected, c.f. [AN1, Proposition 3.11].

The tensor category $\operatorname{Rep}\left(\mathbb{k}_{\tau}^{\sigma} \mathcal{T}\right)$ can be described in terms of the combinatorics of double groupoids, as in [AN1, 3.4]: objects are $\mathcal{H}$-graded vector spaces endowed with a left $\sigma$-twisted action of the vertical composition groupoid $\mathcal{B} \rightrightarrows \mathcal{H}$ by linear isomorphisms.

This means that every $A \in \mathcal{B}$ determines a linear isomorphism $A: V_{b(A)} \rightarrow V_{t(A)}$, compatible with vertical composition in the following sense:

$$
A .(B . v)=\sigma(A, B){ }_{B}^{A} \cdot v,
$$

for all vertically composable $A, B \in \mathcal{B}$, and for all $v \in V_{b(A)}$.

Tensor product is as in $\mathcal{H}$ graded vector spaces, with twisted $\mathcal{V}$-action induced by $\Delta$. The unit object is the target subalgebra $\left(\mathbb{k}_{\tau}^{\sigma} \mathcal{T}\right)_{t}=\oplus_{P \in \mathcal{P}} \mathbb{k}_{P} \mathbf{1}$, with $\mathcal{H}$-grading defined by

$$
\left(\mathbb{k} \mathcal{T}_{t}\right)_{x}= \begin{cases}\mathbb{k}_{P} \mathbf{1}, & \text { if } x=\mathrm{id} P, \\ 0, & \text { if } x \notin \mathcal{P},\end{cases}
$$

for all $x \in \mathcal{H}$, and $\mathcal{B}$-action $A$.P $_{P} \mathbf{1}=\epsilon_{t}\left(A_{P} \mathbf{1}\right)$. 
4.1. The category $\operatorname{Rep}\left(\mathbb{k}_{\tau}^{\sigma} \mathcal{T}\right)$. We aim to give a combinatorial description of the category $\operatorname{Rep}\left(\mathbb{k}_{\tau}^{\sigma} \mathcal{T}\right)$ in terms of the matched pair $(\mathcal{H}, \mathcal{V})$ of groupoids associated to $\mathcal{T}$.

Define the category $\operatorname{Vect}_{\mathcal{V}}^{\mathcal{H}}(\sigma, \tau)$ as follows. Objects in $\operatorname{Vect}_{\mathcal{V}}^{\mathcal{H}}(\sigma, \tau)$ are $\mathcal{H}$-graded vector spaces $M=\oplus_{x \in \mathcal{H}} M_{x}$, together with a right action of $\mathcal{V}$ by linear isomorphisms

$$
g: M_{x} \rightarrow M_{x \triangleleft g}
$$

for all $x \in \mathcal{H}$ such that $r(x)=t(g)$.

That is, for all homogeneous elements $m \in M$ and all composable $g, h \in \mathcal{V}$ such that $r(|m|)=t(g)$, and for any $P \in \mathcal{P}$

$$
\begin{gathered}
m\left\llcorner\operatorname{id}_{P}= \begin{cases}m & \text { if } P=r(|m|) \\
0 & \text { otherwise, }\end{cases} \right. \\
(m\llcorner g)\llcorner h=\sigma(\square g, \quad \square h) \quad m<(g h), \\
\mid m\llcorner g|=| m \mid \triangleleft g .
\end{gathered}
$$

The tensor product of two objects $M=\oplus_{x \in \mathcal{H}} M_{x}, N=\oplus_{y \in \mathcal{H}} N_{y}$ is given by

$$
(M \otimes N)_{z}=\oplus_{x y=z} M_{x} \otimes N_{y},
$$

with right action of $\mathcal{V}$ on homogeneous elements

$$
(m \otimes n)<g=\tau(\stackrel{|m|}{\square}|n| \triangleright g, \quad \square g) \quad m<(|n| \triangleright g) \otimes n<g .
$$

The unit object is $\mathbb{k} \mathcal{P}$ with $\mathcal{H}$-grading determined by $|P|=\operatorname{id}_{P}, P \in \mathcal{P}$, and right $\mathcal{V}$-action given by $P<g=b(g)$, for all $P \in \mathcal{P}, g \in \mathcal{V}$ such that $t(g)=P$.

Proposition 4.2. For any matched pair of groupoids $(\mathcal{H}, \mathcal{V})$ and any pair $(\sigma, \tau) \in$ $\operatorname{Opext}(\mathbb{k} \mathcal{T})$ there is a natural tensor equivalence

$$
\operatorname{Vect}_{\mathcal{V}}^{\mathcal{H}}(\sigma, \tau) \cong \operatorname{Rep}\left(\left(\mathbb{k}_{\tau}^{\sigma} \mathcal{T}\right)^{o p}\right) .
$$

Proof. We will actually prove that $\operatorname{Vect}_{\mathcal{V}}^{\mathcal{H}}(\sigma, \tau)$ is tensor equivalent to the category of right $\mathbb{k}_{\tau}^{\sigma} \mathcal{T}$-modules.

Let $M$ be a right $\mathbb{k}_{\tau}^{\sigma} \mathcal{T}$-module. The $\mathcal{H}$-grading on $M$ is defined by

$$
M_{x}:=M \cdot \frac{x}{\square},
$$

for any $x \in \mathcal{H}$. If $x \in \mathcal{H}, h \in \mathcal{V}$ are elements such that $r(x)=t(h)$, then the action of $h$ on $m \in M_{x}$ is given by

$$
m<h:=m \cdot \stackrel{x}{\square} h .
$$


Straightforward calculations show that equations (4.8), (4.9), (4.10) and (4.11) are fulfilled.

Conversely, assume that $N=\oplus_{y \in \mathcal{H}} N_{y}$ is an object in $\operatorname{Vect}_{\mathcal{V}}^{\mathcal{H}}(\sigma, \tau)$. Define

$$
n \cdot \square g:=\left\{\begin{array}{ll}
n<g & \text { if } y=x \\
0 & \text { otherwise, }
\end{array} \quad \forall n \in N_{y}, \quad \square g \in \mathcal{B} .\right.
$$

This defines a right action and the action on a tensor product is given by the comultiplication of $\mathbb{k}_{\tau}^{\sigma} \mathcal{T}$. Moreover, the above functors are strict tensor functors and define inverse tensor equivalences of categories. Details are left to the reader.

\section{5. $\operatorname{Rep}\left(\mathbb{k}_{\tau}^{\sigma} \mathcal{T}\right)$ as a category of bimodules}

The main goal of this section is to prove that the category $\operatorname{Rep}\left(\mathbb{k}_{\tau}^{\sigma} \mathcal{T}\right)$ is grouptheoretical. To achieve this we shall use an auxiliary tensor category, namely the category $\mathcal{C}(\mathcal{D}, \omega, \mathcal{V})$ introduced in Section 2 .

Along this section $\mathcal{D} \rightrightarrows \mathcal{P}$ will be the diagonal groupoid $\mathcal{D}=\mathcal{V} \bowtie \mathcal{H}$ associated to the matched pair as in Section 3. We do not assume however that the wide subgroupoid $\mathcal{V} \rightrightarrows \mathcal{P}$ is connected.

We begin with the following technical lemma. For any $P \in \mathcal{P}$ let

$$
\theta(P)=\#\{g \in \mathcal{V}: \mathfrak{s}(g)=P\}=\#\{g \in \mathcal{V}: \mathfrak{e}(g)=P\} .
$$

Observe that $\theta(P)$ is constant on the connected components of $\mathcal{P}$ with respect to $\mathcal{V}$.

Let us define elements $\Lambda_{P}, \widetilde{\Lambda}_{P}, \Lambda$ in the groupoid algebra $\mathbb{k} \mathcal{V}$ as follows

$$
\Lambda_{P}=\frac{1}{\theta(P)} \sum_{g \in \mathcal{V}: \mathfrak{s}(g)=P} g, \quad \widetilde{\Lambda}_{P}=\frac{1}{\theta(P)} \sum_{g \in \mathcal{V}: \mathfrak{e}(g)=P} g, \quad \Lambda=\sum_{P \in \mathcal{P}} \Lambda_{P} .
$$

Note that also $\Lambda=\sum_{P \in \mathcal{P}} \widetilde{\Lambda}_{P}$.

Lemma 5.1. The following identities hold, for all $h \in \mathcal{V}$ :
(i) $h \Lambda_{P}=\left\{\begin{array}{l}\Lambda_{\mathfrak{s}(h)} \\ 0\end{array}\right.$
if $\mathfrak{e}(h)=P$
otherwise,
and $\widetilde{\Lambda}_{P} h=\left\{\begin{array}{l}\widetilde{\Lambda}_{\mathfrak{e}(h)} \\ 0\end{array}\right.$
if $\mathfrak{s}(h)=P$
otherwise,
(ii) $h \Lambda=\operatorname{id}_{\mathfrak{s}(h)} \Lambda$,
(iii) $\Lambda h=\Lambda \operatorname{id}_{\mathfrak{e}(h)}$.

Proof. Straightforward.

For any left $\mathbb{k} \mathcal{V}$-module $M$ we denote

$$
\mathcal{V}_{M}:=\left\{m \in M: g \rightarrow m=\mathrm{id}_{\mathfrak{s}(g)} \rightarrow m \text { for any } g \in \mathcal{V}\right\} .
$$

Remark 5.2. Note that for $M \in \mathcal{C}(\mathcal{D}, \omega, \mathcal{V})$, we have $\mathcal{V}_{M}=\Lambda \rightarrow M$. Indeed $\Lambda \rightarrow$ $M \subseteq \mathcal{V}_{M}$ follows from Lemma 5.1 (ii), and if $m \in \mathcal{V}_{M}$ then

$$
\Lambda \rightarrow m=\sum_{P \in \mathcal{P}} \frac{1}{\theta(P)} \sum_{\mathfrak{s}(g)=P} g \rightarrow m=\sum_{P \in \mathcal{P}} \operatorname{id}_{P} \rightarrow m=m .
$$

We now state the main result of this section. 
Theorem 5.2. Let $(\sigma, \tau) \in \operatorname{Opext}(\mathbb{k} \mathcal{T})$ and let $\omega=\omega(\sigma, \tau)$ be the 3-cocycle defined by (4.4). Then the categories $\operatorname{Rep}\left(\mathbb{k}_{\tau}^{\sigma} \mathcal{T}\right)$ and $\mathcal{C}(\mathcal{D}, \omega, \mathcal{V})$ are tensor equivalent.

Proof. Set $p: \mathcal{D} \rightarrow \mathcal{H}, p(g, x)=x$ and $\pi: \mathcal{D} \rightarrow \mathcal{V}, \pi(g, x)=g$. Define the functors $\Phi: \operatorname{Vect}_{\mathcal{V}}^{\mathcal{H}}(\sigma, \tau) \rightarrow \mathcal{C}(\mathcal{D}, \omega, \mathcal{V})$, and $\Psi: \mathcal{C}(\mathcal{D}, \omega, \mathcal{V}) \rightarrow \operatorname{Vect}_{\mathcal{V}}^{\mathcal{H}}(\sigma, \tau)$ as follows: $\Phi(W):=\mathbb{k} \mathcal{V} \otimes W$, where the tensor product is the tensor product in $\mathcal{C}(\mathcal{D}, \omega)$.

The $\mathcal{V}$-actions and $\mathcal{D}$-grading are determined by

$$
\begin{gathered}
g \rightarrow(h \otimes w):=g h \otimes w, \quad(h \otimes w)<g:=h(|w| \triangleright g) \otimes w<g, \\
|h \otimes w|:=h|w|,
\end{gathered}
$$

for any homogeneous element $w \in W$ and $g, h \in \mathcal{V}$ appropriately composable.

Given $M \in \mathcal{C}(\mathcal{D}, \omega, \mathcal{V})$ define $\Psi(M):={ }^{\mathcal{V}} M$. The $\mathcal{H}$-grading on $\Psi(M)$ is given by

$$
\|m\|:=p(|m|),
$$

and the right action of $\mathcal{V}$ is the action on $M$ as an object in $\mathcal{C}(\mathcal{D}, \omega, \mathcal{V})$. Let us prove that this functors are well defined. Let $W \in \operatorname{Vect}_{\mathcal{V}}^{\mathcal{H}}(\sigma, \tau)$. Identities (2.3), (2.4), (2.5), (2.7) and (2.8) are easily checked. Let $w \in W, h, g, f \in \mathcal{V}$ appropriately composable, then

$$
\begin{aligned}
((h \otimes w)<g)<f & =h|w| \triangleright g \otimes(w<g)<f \\
& =h(|w| \triangleright g)(|w| \triangleleft g) \triangleright f \otimes(w<g)<f \\
& =\omega(|w|, g, f) h(|w| \triangleright g f) \otimes w<g f,
\end{aligned}
$$

the last identity by (4.9) and (4.7). This proves identity (2.6), thus $\Phi$ is well defined.

We claim that the functors $\Phi, \Psi$ give an equivalence of categories.

Let $\Lambda$ be the element defined by (5.1). Define the map

$$
\gamma_{M}: M \rightarrow \Phi(\Psi(M)), \gamma_{M}(m)=\pi(|m|) \otimes \Lambda \rightarrow m
$$

for any homogeneous element $m \in M$.

The map $\gamma_{M}$ is a $\mathbb{k} \mathcal{V}$-bimodule map: indeed, if $g \in \mathcal{V}$ and $m \in M$ is homogeneous of degree $(h, x) \in \mathcal{D}$ then

$$
\begin{aligned}
\gamma_{M}(g \rightarrow m) & =\pi(g|m|) \otimes \Lambda \rightarrow(g \rightarrow m)=g \pi(|m|) \otimes \Lambda g \rightarrow m \\
& =g \pi(|m|) \otimes \Lambda \operatorname{id}_{\mathfrak{e}(g)} \rightarrow m=g \rightarrow \gamma_{M}(m) .
\end{aligned}
$$

The third equality by Lemma 5.1 (iii). On the other hand

$$
\begin{aligned}
\gamma_{M}(m\llcorner g) & =\pi(|m| g) \otimes \Lambda \rightarrow(m\llcorner g) \\
& =h(|m| \triangleright g) \otimes(\Lambda \rightarrow m) \leftarrow g=\gamma_{M}(m)\llcorner g .
\end{aligned}
$$

The inverse of $\gamma_{M}$ is given by $\bar{\gamma}_{M}(g \otimes \Lambda \rightarrow m)=g h^{-1} \rightarrow m$, if $m \in M$ is homogeneous of degree $(h, x)$.

Claim 5.1. The map $\bar{\gamma}_{M}$ is well defined.

Proof. First note that if $m \in M$ is an homogeneous element of degree $(h, x)$ then the homogeneous component of degree (id, $x$ ) of $\Lambda_{\mathfrak{s}(h)} \rightarrow m$ is $h^{-1} \rightarrow m$. 
Now, let $m, n \in M$ be homogeneous elements of degree $(h, x)$ and $\left(h^{\prime}, x^{\prime}\right)$ respectively, such that $\Lambda \rightarrow m=\Lambda \rightarrow n$. Equation (2.5) implies that $\Lambda_{\mathfrak{s}(h)} \rightarrow m=\Lambda_{\mathfrak{s}\left(h^{\prime}\right)} \rightarrow$ $n$. Any homogeneous component of $\Lambda_{\mathfrak{s}(h)} \rightarrow m$, respectively $\Lambda_{\mathfrak{s}\left(h^{\prime}\right)} \rightarrow n$, has degree $(g h, x)$, resp. $\left(g h^{\prime}, x^{\prime}\right)$, for some $g \in \mathcal{V}$. This implies that $x=x^{\prime}$. By the previous observation $h^{-1} \rightarrow m=h^{\prime-1} \rightarrow n$.

If $W \in \operatorname{Vect}_{\mathcal{V}}^{\mathcal{H}}(\sigma, \tau)$ then $\Psi(\Phi(W))=^{\mathcal{V}}(\mathbb{k} \mathcal{V} \otimes W) \cong W$, the isomorphism $\phi_{W}$ : $W \longrightarrow \mathcal{V}(\mathbb{k} \mathcal{V} \otimes W)$, being given by $\phi(w):=\Lambda \mathrm{id}_{\mathfrak{e}(|w|)} \otimes w$, for any homogeneous element $w \in W$.

Finally, we shall prove that $\Phi$ is a tensor functor. Let $W, U \in \operatorname{Vect}_{\mathcal{V}}^{\mathcal{H}}(\sigma, \tau)$ and define $\xi_{W U}: \Phi(W \otimes U) \rightarrow \Phi(W) \bar{\otimes} \Phi(U)$ in the form

$$
\xi_{W U}(g \otimes(w \otimes u)):=(g \otimes w) \bar{\otimes}(\mathfrak{e}(|w|) \otimes u),
$$

for any appropriate choice of $g \in \mathcal{V}$, and homogeneous $w \in W, u \in U$.

The natural map $\xi_{W U}$ is an isomorphism, its inverse $\bar{\xi}_{W U}: \Phi(W) \bar{\otimes} \Phi(U) \rightarrow$ $\Phi(W \otimes U)$ being given by

$$
\bar{\xi}_{W U}((h \otimes z) \otimes(f \otimes y))=h(|z| \triangleright f) \otimes z<f \otimes y .
$$

We next show that $\xi_{W U}$ is a morphism of $\mathcal{V}$-bimodules.

Let $h, g \in \mathcal{V}, w \in W, U \in U$. To prove right $\mathcal{V}$-linearity we compute

$$
\begin{aligned}
& \xi_{W U}((g \otimes(w \otimes u))<h)=\xi_{W U}(g(|w||u| \triangleright h) \otimes(w \otimes u)<h) \\
& =\tau\left(\square^{|w|}|u| \triangleright h, \stackrel{|u|}{\square} h\right) \xi_{W U}(g(|w||u| \triangleright h) \otimes(w \leftarrow(|u| \triangleright h) \otimes u<h)) \\
& =\tau\left(\square^{|w|}|u| \triangleright h, \square^{|u|} h\right)(g(|w||u| \triangleright h) \otimes w<(|u| \triangleright h)) \bar{\otimes}(\mathfrak{s}(|u|) \otimes u<h) \\
& =\tau\left(\square^{|w|}|u| \triangleright h, \square h\right)((g \otimes w) \leftarrow(|u| \triangleright h)) \bar{\otimes}(\mathfrak{s}(|u|) \otimes u<h) \\
& =\tau\left(\square^{|w|}|u| \triangleright h, \square^{|u|} h\right)(g \otimes w) \bar{\otimes}(|u| \triangleright h \otimes u<h) \\
& =\omega(g|w|,|u|, h)(g \otimes w) \bar{\otimes}(|u| \triangleright h \otimes u<h) \\
& =\xi_{W U}(g \otimes(w \otimes u))<h .
\end{aligned}
$$

The fifth equality by (2.9), the sixth follows from the definition of $\omega$, and the last equality follows from equation (2.10). Left $\mathcal{V}$-linearity is similarly established.

Claim 5.2. For all $U, V, W \in \operatorname{Vect}_{\mathcal{V}}^{\mathcal{H}}(\sigma, \tau)$, we have

$$
a_{\Phi(U), \Phi(V), \Phi(W)}\left(\xi_{U, V} \otimes \mathrm{id}\right) \xi_{U \otimes V, W}=\left(\mathrm{id} \otimes \xi_{V, W}\right) \xi_{U, V \otimes W} \Phi\left(a_{U, V, W}\right) .
$$

Proof. Let $U, V, W \in \operatorname{Vect}_{\mathcal{V}}^{\mathcal{H}}(\sigma, \tau), g \in \mathcal{V}$ and let $u \in U, v \in V, w \in W$ be homogeneous elements with appropriately composable degree. The left hand side of this equation evaluated in $g \otimes u \otimes v \otimes w$ gives

$$
\begin{aligned}
a\left(\xi_{U, V} \otimes \mathrm{id}\right) & (g \otimes(u \otimes v) \bar{\otimes} \mathfrak{s}(|w|) \otimes w)=a((g \otimes u \bar{\otimes} \mathfrak{s}(|v|) \otimes v) \bar{\otimes} \mathfrak{s}(|w|) \otimes w) \\
& =\omega(g|u|,|v|,|w|) g \otimes u \bar{\otimes}(\mathfrak{s}(|v|) \otimes v \bar{\otimes} \mathfrak{s}(|w|) \otimes w) \\
& =g \otimes u \bar{\otimes}(\mathfrak{s}(|v|) \otimes v \bar{\otimes} \mathfrak{s}(|w|) \otimes w) .
\end{aligned}
$$


The last equality by (4.6). The right hand side of (5.2) evaluated in $g \otimes u \otimes v \otimes w$ gives

$$
\begin{aligned}
\left(\mathrm{id} \otimes \xi_{V, W}\right) \xi_{U, V \otimes W}(g \otimes u \otimes(v \otimes w)) & =\left(\operatorname{id} \otimes \xi_{V, W}\right)(g \otimes u \bar{\otimes} \mathfrak{s}(|v|) \otimes(v \otimes w)) \\
& =g \otimes u \bar{\otimes}(\mathfrak{s}(|v|) \otimes v \bar{\otimes} \mathfrak{s}(|w|) \otimes w) .
\end{aligned}
$$

The proof of the theorem is now complete.

We now can state the main result of this section.

Theorem 5.3. Suppose that $\operatorname{Rep}\left(\mathbb{k}_{\tau}^{\sigma} \mathcal{T}\right)$ is a fusion category. Let $\mathcal{D}$ be the diagonal groupoid associated to $\mathcal{T}$, and let $\omega=\omega(\sigma, \tau) \in H^{3}\left(\mathcal{D}, \mathbb{k}^{\times}\right)$be the 3-cocycle given by (4.4). Then there is an equivalence of tensor categories

$$
\operatorname{Rep}\left(\mathbb{k}_{\tau}^{\sigma} \mathcal{T}\right) \simeq \mathcal{C}(D, \bar{\omega}, V)
$$

where $\bar{\omega}$ is a normalized 3-cocycle on $D$ cohomologous to the restriction $\widehat{\omega}$.

In particular, the category $\operatorname{Rep}\left(\mathbb{k}_{\tau}^{\sigma} \mathcal{T}\right)$ is group-theoretical.

Proof. By [AN1, Proposition 3.11], the assumption implies that $\mathcal{V} \rightrightarrows \mathcal{P}$ is connected. Combining Corollary 2.6 and Theorem 5.2, we get tensor equivalences

$$
\operatorname{Rep}\left(\mathbb{k}_{\tau}^{\sigma} \mathcal{T}\right) \simeq \mathcal{C}(\mathcal{D}, \omega, \mathcal{V}) \simeq \mathcal{C}(D, \widehat{\omega}, V, \widehat{\psi}),
$$

where $\psi \in H^{2}\left(\mathcal{V}, \mathbb{k}^{\times}\right)$is determined by (2.12), and $\widehat{\psi}$ is the 2-cocycle on $V$ obtained by restriction. By [N2, Remark 3.2], there exists a 3 -cocycle $\bar{\omega}$ on $D$ which is cohomologous to $\widehat{\omega}$ and such that $\mathcal{C}(D, \widehat{\omega}, V, \widehat{\psi}) \simeq \mathcal{C}(D, \bar{\omega}, V)$. This finishes the proof of the theorem.

It follows that $\operatorname{Rep}\left(\mathbb{k}_{\tau}^{\sigma} \mathcal{T}\right)$ is the representation category of certain (unique up to gauge equivalence) semisimple finite-dimensional quasi-Hopf algebra. We point out that an explicit description (up to twist equivalence) of this quasi-Hopf algebra has been given in [N2], where the Frobenius-Schur indicators of a group-theoretical fusion category were also computed.

By specializing the results of Ostrik on classification of module categories over group-theoretical categories, we can parameterize module categories and fiber functors for the categories $\operatorname{Rep}\left(\mathbb{k}_{\tau}^{\sigma} \mathcal{T}\right)$.

\section{References}

[AM] N. Andruskiewitsch and J.M. Mombelli, Examples of weak Hopf algebras arising from vacant double groupoids, Nagoya Math. J. 181 (2006) 1-27. Preprint math.QA/0405374.

[AN1] N. Andruskiewitsch and S. Natale, Double categories and quantum groupoids, Publ. Mat. Urug. 10 (2005) 11-51. Preprint math.QA/0308228.

[AN2] - Tensor categories attached to double groupoids, Adv. Math. 200 (2006), no. 2, 539583. Preprint math.QA/0408045.

[DPR] R. Dijkgraaf, V. Pasquier and P. Roche, Quasi-quantum groups related to orbifold models, Modern Quantum Field Theory, Tata Institute, Bombay (1990), World Sci Publ., River Edge, NJ, (1991) 375-383.

[E] C. Ehresmann, Catégories doubles et catégories structurées, C. R. Acad. Sci. Paris 256 (1963) 1198-1201.

[ENO] P. Etingof, D. Nikshych and V. Ostrik, On fusion categories, Ann. of Math. 162 (2005), no. 2, 581-642, Preprint math.QA/0203060.

[M] K. Mackenzie, Double lie algebroides and second-order geometry, I, Adv. Math. 94 (1992) $180-239$. 
[N1] S. Natale, On group theoretical Hopf algebras and exact factorizations of finite groups, J. Algebra 270 (2003), no. 1, 199-211. Preprint math.QA/0208054.

[N2] _ Frobenius-Schur indicators for a class of fusion categories, Pacific J. Math. 221 (2005), no. 2, 353-378. Preprint math.QA/0312466.

[O1] V. Ostrik, Module categories, weak Hopf algebras and modular invariants, Transform. Groups 8 (2003), no.2, 177-206. Preprint math.QA/0111139.

[O2] Module categories over the Drinfeld double of a finite group, Int. Math. Res. Not. 27 (2003), no. 27, 1507-1520. Preprint math.QA/0202130.

[Sch] P. Schauenburg, Hopf imodules, coquasibialgebras, and an exact sequence of Kac, Adv. Math. 165 (2002), no. 2, 194-263

Facultad de Matemática, Astronomía y Física

Universidad NACIONAL DE CóRdoba

CIEM - CONICET

Medina Allende s/N

(5000) Ciudad Universitaria, Córdoba, Argentina

E-mail address: mombelli@mate.uncor.edu

URL: http://www.mate.uncor.edu/mombelli

E-mail address: natale@mate.uncor.edu

URL: http://www.mate.uncor.edu/natale 